\title{
Imitation of a two-action sequence by pigeons
}

\author{
NAM H. NGUYEN, EMILY D. KLEIN, and THOMAS R. ZENTALL \\ University of Kentucky, Lexington, Kentucky
}

\begin{abstract}
Developmental psychologists have described imitation as a process that suggests perspective-taking abilities. However, imitative behavior has been found in animals, which are generally not considered capable of taking the perspective of another. Previous studies with birds have demonstrated the imitation of a single response (sometimes referred to as action-level imitation). In the present experiment, we examined the extent to which pigeons would imitate an unfamiliar sequence of two behaviors (sometimes referred to as program-level imitation). Our results indicate that, although there are individual differences, pigeons show a significant tendency to match a demonstrated sequence of behavior involving, first, a response to a treadle (pecking at it or stepping on it) and, second, pushing aside a screen that blocks access to food (a left-vs.-right push).
\end{abstract}

Imitation is considered an important indicator of human cognitive development (Piaget, 1962). Until recently, reports of imitative learning by animals have been questioned because simpler mechanisms could be proposed to account for the findings (see Zentall, 2003). For example, if the behavior of a demonstrator draws attention to a location or object, it may increase the likelihood that an observer will move to that location (local enhancement) or object (stimulus enhancement) and then by chance make the appropriate response. Lorenz (1935/ 1970), for example, found that ducks were more likely to escape from a pen if they had previously observed another duck escaping through a hole in the fence. However, attention drawn to the hole in the fence, rather than imitation of the demonstrator duck's behavior, may provide the simplest account of the observer ducks' behavior.

Dawson and Foss (1965) first described a design to study imitation in animals while controlling for enhancement effects as well as other simpler motivational, perceptual, and learning factors. In their study, budgerigars (Melopsittacus undulates) learned to remove the lid of a food container in one of three distinctive ways (twisting it to the side with their beak, pushing it away from themselves with their beak, or pulling it toward themselves with their foot). When observers that had watched the lid removal were given access to the covered container, they removed the lid in the way they had seen it removed. This experiment demonstrates that observers can learn not just to remove the lid, but also how to remove it.

A variant of Dawson and Foss's (1965) procedure, known as the two-action method, showed imitative learning in both Japanese quail (Coturnix japonica; Akins \& Zentall, 1996, 1998; Dorrance \& Zentall, 2001) and pigeons (Columba livia; Kaiser, Zentall, \& Galef, 1997;

Correspondence should be addressed to T. R. Zentall, Department of Psychology, University of Kentucky, Lexington, KY 40506 (e-mail: zentall@uky.edu).
Zentall, Sutton, \& Sherburne, 1996). In these experiments, birds observed a demonstrator either peck at or step on a treadle for food reinforcement. When tested, the observer birds made the response that they had observed with high probability. This task can be described as moving a manipulandum in the same way with two different parts of the body.

A variation of the two-action method, known as the bidirectional control procedure, was developed by Heyes and Dawson (1990). In their study, demonstrator rats were trained to push an overhead pole either to the left or right. After observation, observer rats showed a significant tendency to push the pole in the same direction that they had seen it pushed. In contrast to the two-action method, this task can be described as moving a manipulandum in two different ways with the same part of the body. Later research, however, suggested that the direction that observers pushed the pole may have been influenced by odor cues left by the demonstrators on the side of the pole that they pushed, rather than by the visual observation of the demonstrators (Mitchell, Heyes, Gardner, \& Dawson 1999).

Akins, Klein, and Zentall (2002) modified this procedure to test for imitation in Japanese quail, a species less likely to be influenced by odor cues. In their design, observer quail viewed a demonstrator push a feeder-blocking screen to the left or right of a feeder opening. When tested, the observer quail showed a significant tendency to push the screen in the same direction as their demonstrator. Furthermore, when control observers saw the screen move without being pushed by the demonstrator (a control for object-movement reenactment or learned affordances), they did not match the direction of screen movement (see also Klein \& Zentall, 2003, for inclusion of a control for possible odor cues). Thus, the bidirectional control procedure appears to provide a valid alternative procedure for demonstrating imitation in birds.

In the imitation literature, a distinction has been made between the imitation of a single action and that of a se- 
quence of actions (sometimes referred to as programlevel imitation; Byrne, 2002). For example, Barr, Dowden, and Hayne (1996) found that 6- and 12-month-old infants are capable of imitating a single action but show little evidence of imitating the second or third action in a sequence even when they are physically able to perform each individual action. Although there is some evidence that 1-year-olds can accurately imitate a two-action sequence (Bauer \& Mandler, 1992; Mandler \& McDonough, 1995), the procedures involved prior practice with two-action sequences, encouragement to imitate, and a verbal description to the children of the actions that were modeled. Thus, the ability to imitate a sequence of actions, at least in younger children, appears to require instruction with reinforcement for the completion of the sequence to be designated as "correct."

Free-ranging gorillas (Byrne \& Russon, 1998) and orangutans (Russon \& Galdikas, 1993) have been reported to imitate complex sequences of behavior. However, these reports are largely anecdotal in nature and do not include necessary controls. Evidence for imitation of a sequence of actions by an animal in a laboratory setting has been reported by Whiten (1998), who observed that chimpanzees, given sufficient training, can perform a set of three actions to open an "artificial fruit" and obtain a food reward. Imitation of an action sequence has been referred to as program-level imitation because it requires the organization of actions into a logical sequence (Byrne, 2002).

Howard and White (2003) examined the imitation of a two-action sequence by pigeons. One action was stepping onto a box. The second was pecking a response key. However, after observing a demonstrator, observers showed little evidence of imitating the sequence, even after they had been explicitly trained by the experimenter to perform one of its elements - to peck at the response key when the floor was raised.

The purpose of the present study was to determine whether pigeons would be able to imitate a sequence of two actions to obtain reinforcement. The two-response sequence was a combination of the two-action method involving the pecking and stepping responses used by Akins and Zentall (1996) and the bidirectional-control procedure used by Akins et al. (2002). First, demonstrator pigeons were required to either peck at or step on a treadle. The treadle response raised the feeder but a screen in front of the feeder opening prevented the pigeon from eating. Second, to obtain food, the pigeon was required to push the screen to the left or right. Each observer was then exposed to a demonstrator performing one of four behavioral sequences: treadle peck/screen push left, treadle peck/screen push right, treadle step/ screen push left, or treadle step/screen push right. The observer was then given access to the treadle and screen and could obtain food by performing any one of the four sequences. If the observers tended to match the sequence demonstrated, it would indicate that they are capable of imitating a two-action sequence (program-level imitation).

\section{METHOD}

\section{Subjects}

Twenty-two adult White Carneaux pigeons (Columba livia) of undetermined sex served as subjects. Four pigeons were trained as demonstrators (these pigeons had previously served as observers in a social learning experiment), and the remaining pigeons served as observers. All of the pigeons were purchased as retired breeders from the Palmetto Pigeon Plant (Sumter, SC). Ten of the observers previously had been trained on an unrelated visual discrimination, and the remaining observers were experimentally naive. The pigeons were individually housed in vented cages located in a colony room on a 12:12- h light:dark cycle. They were placed on a restricted food diet and were maintained at $80 \%-85 \%$ of their freefeeding weights throughout the experiment. Water and grit were freely available in the pigeons' home cages.

\section{Apparatus}

The apparatus used in the present experiment (see Figure 1) combined features from the apparatus used in similar imitative learning studies involving Japanese quail (Akins et al., 2002; Akins \& Zentall, 1996). It consisted of two large modular chambers (Colbourn Instruments, Model H10-11R-TC, Lehigh Valley, PA) placed side by side. Each chamber measured $30.5 \times 25.5 \times 28.0 \mathrm{~cm}$. The common sidewall of the two chambers was made of transparent Plexiglas, allowing observers to view demonstrator responses in the adjacent chamber. A speaker attached to the front wall of the demonstrator's chamber provided white noise, which masked extraneous noise while the pigeons were in either chamber.

The demonstrator chamber contained a response treadle $(3.8 \times$ $3.8 \mathrm{~cm}$ ) attached to the front panel and located $1.0 \mathrm{~cm}$ from the wall between the two chambers and $2.0 \mathrm{~cm}$ from the floor. A microswitch attached to the treadle operated the feeder whenever the treadle was depressed. A rectangular feeder opening was located on the front wall of the demonstrator's chamber. The opening could be covered with a 7.6- $\times 5.0-\mathrm{cm}$-wide wire screen. Wires on the screen were spaced apart $1.6 \mathrm{~cm}$ horizontally and $2.5 \mathrm{~cm}$ vertically. The screen could be slid either to the left or right along tracks located above and below the feeder opening. A stiff wire, attached to the screen on the right side, protruded outside the demonstrator chamber, allowing the experimenter to push or pull the screen along the track. To prevent the demonstrator from pushing the screen in an unassigned direction, a stop was placed along the wire (outside the chamber for right-pushing demonstrators, inside the chamber for left-pushing demonstrators) during training and during the observation period of testing. The stop was removed when observer pigeons were tested, allowing the screen to move in either direction. The subjects were given $2.0 \mathrm{sec}$ access to food (Purina Pro Grains) when the feeder tray was raised and illuminated by a feeder light. The demonstrator chamber was continuously illuminated by a white houselight ( $28 \mathrm{~V}, 0.1 \mathrm{~A}$ ) mounted on the front panel near the chamber's ceiling.

The observer's chamber was similar to the demonstrator's chamber, but it contained no houselight, treadle, or feeder. The lack of illumination within the observer's chamber allowed easier observation of the demonstrator's responses.

\section{Procedure}

Demonstrator training. Demonstrators were feeder trained in the demonstrator's chamber and habituated to the presence of the experimenter. The pigeons were then shaped to either peck at $(n=2)$ or step on $(n=2)$ the treadle. Whether a response was a peck or a step was judged by the experimenter, but the nature of the apparatus and the pigeon's body made these responses mutually exclusive (i.e., the pigeon had to lift its head and pull it back to step on the treadle, whereas it had to step back from the treadle to peck at it). Each successful response raised and illuminated the feeder, allow- 


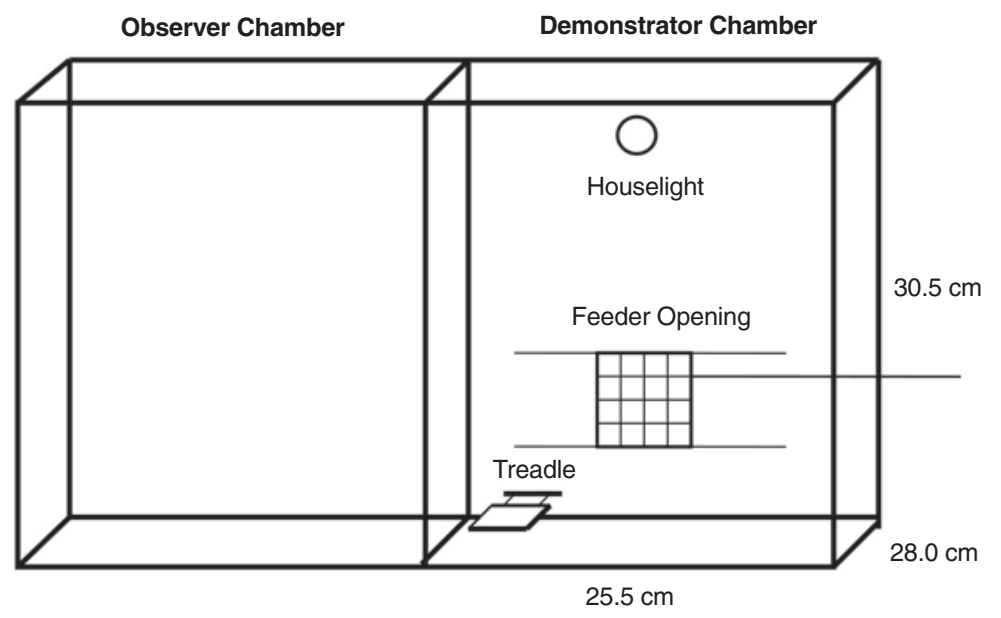

Figure 1. A schematic of the apparatus used in the experiment.

ing the pigeon to eat for $2.0 \mathrm{sec}$. The reinforcement duration began when a photobeam, located within the feeder opening, was broken by the pigeon's head. After a demonstrator reliably stepped on or pecked at the treadle, a wire-mesh screen was introduced in front of the feeder opening. Two demonstrators, one each from the pecking and stepping conditions, were shaped by successive approximations to push the screen to the left using their beak. The remaining pecking and stepping demonstrators were shaped to push the screen to the right. A screen-push response was defined as a push that moved the screen far enough for the pigeon to insert its head into the feeder opening and break the photobeam (which started the 2-sec reinforcement). Thus, each demonstrator was assigned to perform a unique sequence of behavior (treadle peck/screen push left, treadle peck/screen push right, treadle step/screen push left, or treadle step/screen push right).

To reduce the likelihood of the presence of another pigeon disrupting the performance of the demonstrator during the observation period in testing, following acquisition of the response sequence, the demonstrators performed in the presence of a pigeon that was not used as an observer in the experiment.

Observer training. Observers were feeder trained in the demonstrator's chamber and were habituated to the observer's chamber and the presence of the experimenter. While observers were in the demonstrator's chamber, the screen was secured in an open position either to the left or right (which was varied systematically over daily training sessions to provide the observers with equal experience with the screen being positioned to the left and right of the feeder opening). The criterion for successful completion of feeder training for the observer pigeons was 10 sessions in which the observer inserted its head into the feeder opening to obtain reinforcement, 24 times within $10 \mathrm{~min}$.

Testing. The observers were randomly assigned to groups with the provision that each group was assigned either one or two of the experimentally naive pigeons. During testing, an observer was placed in the observer's chamber until its assigned demonstrator successfully completed 60 correct behavioral sequences. After completing the sequences, the demonstrator was removed and the observer was placed into the demonstrator's chamber for $20 \mathrm{~min}$. Each response made by the observer was recorded. The performance of any of the four sequences resulted in reinforcement. Two observers that failed to push the screen in either direction (one that observed a demonstrator peck the treadle and push the screen to the left and one that observed a demonstrator peck the treadle and push the screen to the right; the mean percentage of matching treadle re- sponses for these two pigeons was $65.7 \%$ ), were dropped from the study and replaced by two similarly inexperienced pigeons.

\section{RESULTS}

We used the percentage of matching individual behaviors as a measure of imitation, and chance for the treadle response and the screen push was $50 \%$. Chance for the percentage of matching response sequences was $25 \%$. In all analyses, the differences between experienced and naive birds were examined, but none were found so the data were pooled.

\section{Treadle Response}

The observers varied considerably in the total number of treadle responses made. The mean total treadle responses for the observers was $55.8(S E M=10.7)$, with a range of 4-139 responses. Although the pigeons made more stepping responses $(M=43.0, S E M=10.3)$ than pecking responses $(M=13.0, S E M=8.5)$, this difference was not statistically significant $[t(15)=1.90, p=$ .08]. However, because of this discrepancy, we used the percentage of matching treadle responses (number of matching treadle responses divided by total number of treadle responses $\times 100$ ) for each observer, for all subsequent statistical analyses.

The results indicated a strong tendency for observers to match the behavior of the demonstrator. The mean percentage of matching treadle responses was $66.6 \%$ $(S E M=11.0)$. Because the percentage scores were not normally distributed, the percentages were subjected to an arcsine transformation, which corrects for the upper and lower limits (1.0 and 0 , respectively) placed on percentage scores. The transformed percentage scores were compared with the transformed expected value of $50 \%$, using a $t$ test. The $t$ test indicated that the percentage of matching treadle responses was significantly greater than chance $[t(15)=2.72, p<.02]$. 
Unexpectedly, there was considerable variability in the tendency to match treadle responses among the four groups (see the left side of Figure 2). A two-way analysis of variance (ANOVA) performed on the transformed treadle-response matching scores indicated a significant effect on matching of the observed treadle response [stepping vs. pecking, $F(1,12)=5.02, p<.05]$. However, neither the effect of the direction of screen push $[F(1,12)=$ $1.06]$, nor the treadle-response $\times$ direction-of-screenpush interaction $(F<1)$, was significant. The effect of treadle response can be attributed to the fact that, independent of group, the pigeons showed a bias to step on the treadle $(70.1 \%)$ rather than peck at it $(29.9 \%)$. Thus, they were more likely to match the step response than the peck response.

\section{Screen Push}

The total number of screen pushes made by each observer also varied considerably. The mean total screen pushes for the observers was $44.4(S E=9.19)$, with a range of 1-121. Overall, the pigeons tended to push the screen more often to the right $(M=25.44, S E=8.94)$ than to the left $(M=18.9, S E=5.10)$, although this difference was not statistically significant $[t(15)<1]$. The mean percentage of matching screen pushes was determined for each observer (number of matching screen pushes divided by total number of screen pushes $\times 100$ ). The overall mean percentage of matching screen pushes for the observers was $59.0 \%(S E=10.0)$. When the transformed percentage of matching screen responses was subjected to a statistical test, however, the difference from chance approached, but did not reach, statistical significance $[t(15)=1.88, p=.08]$. A two-way ANOVA performed on the transformed screen-push matching scores for the four counterbalancing groups indicated that neither main effect (direction of screen pushing observed or treadle response observed) nor the interaction was significant (all $F \mathrm{~s}<1.05)$.

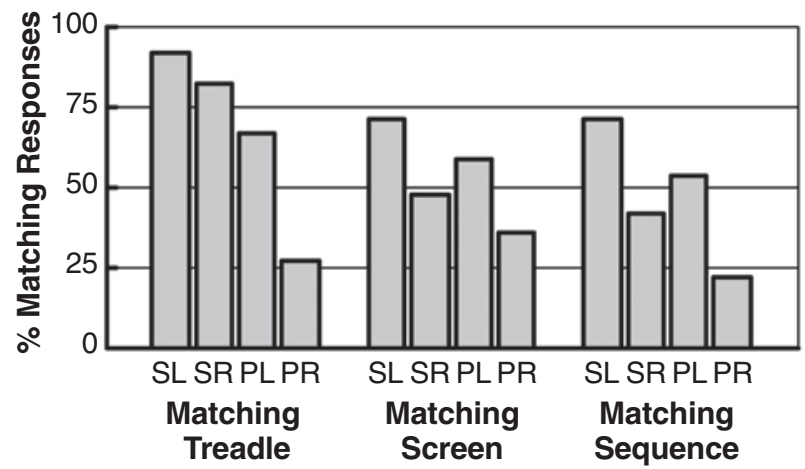

Figure 2. Percentage matching treadle responses (left), matching screen pushes (center), and matching treadle responsescreen-push sequences (right) for pigeons that observed a demonstrator step on the treadle and push the screen to the left (SL), step on the treadle and push the screen to the right (SR), peck the treadle and push the screen to the left (PL), and peck the treadle and push the screen to the right (PR).

\section{Behavioral Sequence}

For purposes of the present experiment, the measure of two-action imitation is the mean percentage of matching sequences relative to total sequences $(47.0 \%, S E=11.0)$. Inspection of the data revealed that 9 of the 16 observers tested obtained mean percentage scores greater than $25 \%$. A binomial test with chance at .25 indicated that this number was statistically different from chance $(p<.02)$. When the transformed percentage score for each pigeon was subjected to statistical analysis and compared with the transformed value of chance $(25 \%)$, the difference was significant $[t(15)=2.36, p<.03]$. A two-way ANOVA performed on the transformed sequence-matching scores for the four counterbalancing groups indicated that neither main effect (direction of screen pushing observed or treadle response observed) nor the interaction was significant (all $F$ s $<2.03$ ).

In addition, there was evidence that pigeons that imitated the screen push also imitated the treadle response. A Pearson product-moment correlation performed on the percentage of matching treadle responses and the percentage of matching screen pushes indicated significant correlation $(r=.44)$. Furthermore, if imitation of the two responses were independent (as would be expected if pigeons did not tend to imitate the sequence of their demonstrator's behavior), the conditional probability of treadle-response matching, given screen-push matching (pigeons for which the percentage of matching responses was above $50 \%$ ) would be expected to be the same as the conditional probability of treadle-response matching, given the absence of screen-push matching (pigeons for which the percentage of matching responses was equal to or below 50\%). However, for pigeons that matched the demonstrator's screen push, the probability that it also matched the demonstrator's treadle response was .83 , whereas for pigeons that did not match the demonstrator's screen push, the probability that it matched the demonstrator's treadle response was only .50 .

\section{DISCUSSION}

The results of the present experiment indicate that the pigeons showed a significant tendency to match the demonstrated treadle response. Evidence for matching the demonstrated direction of screen push, although in the right direction, was not so clear. However, when the treadle response and screen-push data were combined, it was found that the observers produced significantly more matching sequences than would have been expected by chance. Thus, in the present experiment, we found suggestive evidence for imitation of a two-action sequence of the type studied in infants (Bauer \& Mandler, 1992; Mandler \& McDonough, 1995) and chimpanzees (Whiten, 1998). This conclusion is further supported by the correlation between matching treadle responses and matching screen pushes, as well as by the difference between the conditional probability of treadle-response matching, given the presence versus the absence of screenpush matching. Although under the present conditions 
not all of the pigeons imitated the two-action sequence, more of them did than would be expected by chance. To our knowledge, this is the first evidence of two-action sequence imitation in nonhuman animals other than chimpanzees (Whiten, 1998).

In the present experiment, the pigeons had a somewhat greater tendency to step than to peck, independent of the observed response. This bias is probably related to the height of the treadle above the floor. Had the treadle been raised slightly, the bias may have been avoided. In support of this hypothesis, Japanese quail, a considerably smaller bird, showed a stronger tendency to peck than to step in the same apparatus (Akins \& Zentall, 1996, 1998). Given the counterbalancing of treadle response, however, this bias only could have constrained the degree to which the pigeons imitated the treadle response. Thus, in spite of this bias, the pigeons showed a significant tendency to match the sequence of behavior that they had observed.

It is not clear why there was better imitation of the treadle response than of the screen push in the present experiment. It is possible that the observation of a demonstrator moving the treadle with a distinctive part of its body is more detectable than the observation of it moving the screen in a distinctive direction. However, earlier research with this same apparatus found that pigeons demonstrated significant matching of left-right screen pushing (Klein \& Zentall, 2003). In fact, in that study, pigeons tended to match the direction of their demonstrator's screen push $(85.0 \%)$ to the same extent as pigeons in a previous study tended to match their demonstrator's treadle response $(84.2 \%$; Dorrance \& Zentall, 2001).

Alternatively, it is possible that adding the treadle response to the screen-pushing response had a disruptive effect on matching the screen-pushing response. It may be that pigeons have more difficulty organizing a sequence of two behaviors even when they can readily imitate either behavior individually. Thus, when two behaviors are observed, the first may be better remembered and imitated than the second (a primacy effect; see Barr et al., 1996, for a similar effect found in infants). Nevertheless, the results of the present study suggest that pigeons are able to imitate a two-action sequence, a skill that does not appear in human infants until they are at least a year old.

\section{REFERENCES}

AKins, C. K., Klein, E. D., \& Zentall, T. R. (2002). Imitative learning in Japanese quail (Coturnix japonica) using the bidirectional control procedure. Animal Learning \& Behavior, 30, 275-281.

AKIns, C. K., \& Zentall, T. R. (1996). Imitative learning in male Japa- nese quail (Coturnix japonica) involving the two-action method. Journal of Comparative Psychology, 110, 316-320.

AKINS, C. K., \& Zentall, T. R. (1998). Imitation in Japanese quail: The role of reinforcement of demonstrator responding. Psychonomic Bulletin \& Review, 5, 694-697.

BARR, R., Dowden, A., \& HAYNe, H. (1996). Developmental changes in deferred imitation by 6- to 24-month-old infants. Infant Behavior \& Development, 19, 159-170.

Bauer, P. J., \& Mandler, J. M. (1992). Putting the horse before the cart: The use of temporal order in recall of events by one-year-old children. Developmental Psychology, 28, 441-452.

BYRNE, R. W. (2002). Imitation of novel complex actions: What does the evidence from animals mean? Advances in the Study of Behavior, 31, 77-105.

Byrne, R. W., \& Russon, A. E. (1998). Learning by imitation: A hierarchical approach. Behavioral \& Brain Sciences, 21, 667-721.

Dawson, B. V., \& Foss, B. M. (1965). Observational learning in budgerigars. Animal Behaviour, 13, 470-474.

DORRANCE, B. R., \& Zentall, T. R. (2001). Imitative learning in Japanese quail (Coturnix japonica) depends on the motivational state of the observer quail at the time of observation. Journal of Comparative Psychology, 115, 62-67.

Heyes, C. M., \& Dawson, G. R. (1990). A demonstration of observational learning using a bidirectional control. Quarterly Journal of Experimental Psychology, 42B, 59-71.

Howard, M. L., \& White, K. G. (2003). Social influence in pigeons (Columba livia): The role of differential reinforcement. Journal of the Experimental Analysis of Behavior, 79, 175-191.

Kaiser, D. H., Zentall, T. R., \& Galef, B. G., JR. (1997). Can imitation in pigeons be explained by local enhancement together with trialand-error learning? Psychological Science, 8, 459-460.

Klein, E. D., \& ZENTALL, T. R. (2003). Imitation and affordance learning by pigeons (Columba livia). Journal of Comparative Psychology, 117, 355-362.

LorenZ, K. (1970). Companions as factors in the bird's environment. In K. Lorenz (Ed.) \& R. Martin (Trans.), Studies in animal and human behavior (Vol. 1, pp. 101-258). London: Methuen. (Original work published 1935)

MANDLER, J. M., \& MCDonough, L. (1995). Long-term recall of event sequences in infancy. Journal of Experimental Child Psychology, 59, 457-474.

Mitchell, C. J., Heyes, C. M., Gardner, M. R., \& Dawson, G. R. (1999). Limitations of a bidirectional control procedure for the investigation of imitation in rats: Odour cues on the manipulandum. Quarterly Journal of Experimental Psychology: 52B, 193-202.

Piaget, J. (1962). Play, dreams, and imitation in childhood (C. Gattegno \& F. M. Hodgson, Trans.). New York: Norton.

Russon, A. E., \& GALDIKAS, B. M. F. (1993). Imitation in free-ranging rehabilitant orangutans (Pongo pygmaeus). Journal of Comparative Psychology, 107, 147-161.

Whiten, A. (1998). Imitation of the sequential structure of actions by chimpanzees (Pan troglodytes). Journal of Comparative Psychology, 112, 270-281.

Zentall, T. R. (2003). Imitation by animals: How do they do it? Current Directions in Psychological Science, 12, 91-95.

Zentall, T. R., Sutton, J. E., \& Sherburne, L. M. (1996). True imitative learning in pigeons. Psychological Science, 7, 343-346.

(Manuscript received October 30, 2003; revision accepted for publication January 30, 2004.) 